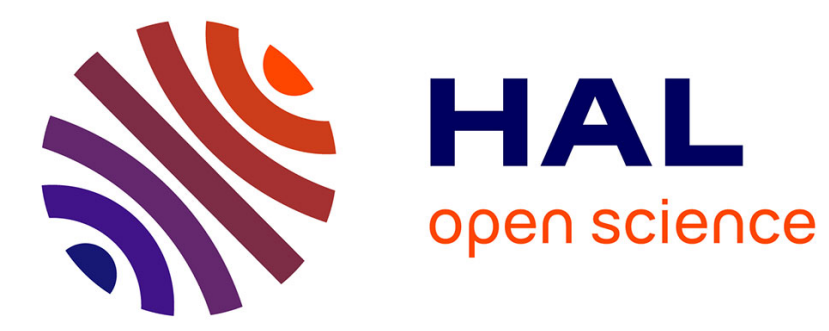

\title{
La gestion durable des forêts tempérées: quelques défis pour le XXIe siècle
}

Christian Barthod

\section{To cite this version:}

Christian Barthod. La gestion durable des forêts tempérées : quelques défis pour le XXIe siècle. Revue forestière française, 1996, 48 (S), pp.235-252. 10.4267/2042/26800 . hal-03444283

\section{HAL Id: hal-03444283 \\ https://hal.science/hal-03444283}

Submitted on 23 Nov 2021

HAL is a multi-disciplinary open access archive for the deposit and dissemination of scientific research documents, whether they are published or not. The documents may come from teaching and research institutions in France or abroad, or from public or private research centers.
L'archive ouverte pluridisciplinaire HAL, est destinée au dépôt et à la diffusion de documents scientifiques de niveau recherche, publiés ou non, émanant des établissements d'enseignement et de recherche français ou étrangers, des laboratoires publics ou privés. 


\title{
LA GESTION DURABLE DES FORÊTS TEMPÉRÉES : QUELQUES DÉFIS POUR LE XXI ${ }^{e}$ SIÈCLE
}

\author{
Ch. BARTHOD
}

La France demeure encore relativement épargnée par les polémiques sur la gestion durable des forêts, qui caractérisent désormais beaucoup de nos partenaires d'Europe centrale et nordique ou d'Amérique du Nord (1). Cette situation particulière s'explique sans doute en partie par la faiblesse des grandes associations de protection de la nature, par le tout petit nombre des scientifiques qui se sont passionnés pour le fonctionnement des écosystèmes forestiers naturels, par le relatif manque d'intérêt pour la forêt d'une société majoritairement d'inspiration culturelle latine, par la prégnance encore revendiquée des références rurales malgré un nombre toujours croissant de générations citadines, sans oublier le fait que les options sylvicoles françaises n'ont offert qu'un nombre relativement limité d'abcès de fixation aux insatisfactions d'une partie, certes minoritaire mais sachant se faire entendre, de la société française.

\section{LA FIN PRÉVISIBLE D'UNE ÉPOQUE}

Les forestiers français auraient néanmoins tort de se réjouir sans nuances de la situation présente, et de croire que le rapport de force assez équilibré qu'elle crée actuellement a vocation à perdurer.

La faiblesse des grandes associations structurées est contrebalancée par un foisonnement de petites associations locales de défense spontanées contre tel ou tel projet. II existe sans doute environ 20000 militants s'intéressant régulièrement ou épisodiquement aux orientations de politique forestière, comme raison principale de leur engagement associatif, ce qui est beaucoup au regard de la situation du milieu associatif français, mais bien peu au regard de la légitimité démocratique. Mais leur audience disproportionnée avec leur effectif montre qu'ils savent toucher une part significative de l'opinion publique, indiquant que celle-ci se reconnaît dans une partie de leurs questions, mais pas forcémenf dans toufes leurs réponses. Au lieu d'avoir à négocier de façon préalable avec un par-

(1) O. TOOLE (R.). - Reforming the Forest Service. - Washington (DC) : Island Press, $1988 .-247 \mathrm{p}$

ROBINSON (G.). - The forest and the trees: a guide to excellent forestry. - Washington (DC) : Island Press, 1988 . - $257 \mathrm{p}$. 


\section{Ch. BARTHOD}

tenaire connu, les forestiers français seront de plus en plus amenés à devoir réagir a posteriori à une contestation d'autant plus virulente de certaines de leurs options qu'elle n'est pas encadrée par une expertise organisée et par des règles de négociation connues de tous. Une difficulté majeure pour les forestiers publics tient à ce qu'ils ne peuvent pas apparaître comme se désolidarisant des conseils municipaux pour lesquels ils exercent les responsabilités découlant de la soumission au régime forestier des forêts publiques. C'est dire que le chemin est étroit, lorsque certains affrontements opposent des élus locaux bénéficiant du suffrage universel et des représentants du monde associatif.

La faiblesse numérique du monde scientifique français s'intéressant aux grands débats sur la biodiversité et la gestion durable des forêts est contrebalancée par la circulation internationale des informations scientifiques, permettant à un nombre croissant de personnalités périphériques au monde forestier traditionnel de s'emparer de grilles d'analyses et de résultats élaborés dans un autre contexte et de les appliquer, sans parfois trop de nuances et de discernement scientifique, aux réalités forestières françaises, sans qu'un véritable débat scientifique public et contradictoire puisse s'établir sur dossiers concrets et enracinés dans un terroir. Par ailleurs un certain manque d'intérêt de trop de forestiers pour les résultats de la recherche internationale sur les questions qui passionnent les militants des associations de protection de la nature n'est pas de nature à permettre l'émergence d'un débat scientifique et technique régulé par les règles épistémologiques de la connaissance. Or il est très dangereux que le débat actuel donne l'impression d'opposer des scientifiques (pas toujours bons connaisseurs des problèmes extérieurs à leur domaine de recherche) et des gestionnaires peu au fait du dernier état des connaissances, même lorsque certains scientifiques ne cachent que très peu leurs engagements militants.

Le manque d'intérêt traditionnel de la société française pour les questions forestières est de plus en plus contrebalancé par l'internationalisation des débats technico-politiques, dont les médias se font normalement l'écho et l'amplificateur, alors même que la structure même de l'expertise administrative française est parfaitement inadaptée à la gestion de ce type de problème, comme l'a montré de façon éclatante la gestion du débat public sur les "pluies acides". A posteriori, les forestiers français ont eu, dans les grandes lignes, majoritairement raison du point de vue scientifique, tout en ayant politiquement tort ${ }^{(2)}$.

L'enracinement culturel revendiqué de notre société dans les valeurs du monde rural s'estompe, au fur et à mesure que la vie citadine façonne la perception désormais majoritaire de la nature, la forêt étant promue, au même titre que les océans, au rang de représentation archétypique de la nature. De plus la médiation que représentait la propriété foncière en milieu rural perd de plus en plus de sa force. D'une part, le monde agricole a établi définitivement sa domination vis-à-vis du propriétaire foncier, ce qui n'est pas motivant pour une forte implication des propriétaires fonciers dans l'avenir du monde rural. D'autre part, avec la disparition progressive de la génération qui a quitté la terre tout en conservant un fort attachement personnel au terroir familial, le morcellement foncier ne peut qu'augmenter et la motivation pour la gestion directe diminuer progressivement. Ceci ne peut que réduire inexorablement le nombre effectif de ceux qui ont à arbitrer par eux-mêmes les divergences inévitables entre le souhaité et le possible dans la gestion particulière d'une propriété. Dans ce contexte, le nombre de ceux qui sont convaincus que l'intérêt général tel qu'ils le conçoivent doit l'emporter sur une conception du droit de propriété qu'ils considèrent comme dépassée ne peut que monter. II existe de fait une demande encore floue pour une appropriafion collective de la forêt, d'autant plus déstabilisante que la forêt publique est minoritaire en France et que le libre accès à toutes les forêts semble un droit légitime à beaucoup de citadins.

(2) BARTHOD (Ch.) et al. - Écologie, débat public et politiques techniques: le dépérissement des forêts dix ans après. - Revue forestière française, vol. XLV, $\mathrm{n}^{\circ} 5,1993, \mathrm{pp} .509-524$ 
Enfin, les forestiers ne devraient pas sous-estimer la puissance symbolique de certains conflits dans lesquels ils sont parties prenantes, sans être toujours à même de contrôler la situation ou même d'en percevoir toujours tous les enjeux, au-delà de ce qui est contesté verbalement : le choc des perceptions et des traditions forestières relevant des cultures latine et germanique, plus manifeste en Alsace, tout en étant bien présent dans d'autres régions de l'Est de la France; la peur des régions en perte de vitesse économique devant la forêt envahissante et la désertification humaine qui la précède et l'accompagne, dans le Morvan ou certaines vallées vosgiennes; le conflit ancien d'appropriation entre ceux qui veulent une forêt contemplée et ceux qui ne se posaient plus assez de questions sur la légitimité des cicatrices de leurs actions dans une forêt gérée, à Fontainebleau; le conflit entre la gratuité de la vie menacée et ce qui est perçu comme une vision prioritairement économique de la nature, dans les affaires de l'Ours ou du Grand Tétras.

\section{LA FORESTERIE FRANÇAISE DANS UN CONTEXTE EN ÉVOLUTION}

Pour toutes les raisons évoquées précédemment, les forestiers français doivent s'interroger sur les stratégies encore possibles dans ce débat sur la gestion durable des torêts. Comme c'est également le cas pour les agriculteurs, même si les transferts financiers de la collectivité ne sont pas du tout du même ordre de grandeur, la sensibilité du secteur forestier aux priorités et aux valeurs de la société vient en effet de sa dépendance vis-à-vis des politiques publiques d'aides, sous forme de subventions et plus encore sous forme d'avantages fiscaux, quelle que soit la légitimité incontestable de ces aides compensatrices de handicaps.

Un peu plus tard que les agriculteurs, les forestiers (privés et publics) découvrent que l'évolution socio-économique et culturelle de la société, la concurrence internationale et la technologie sont en train de modifier radicalement le regard du citoyen et du consommateur sur la forêt et le bois. Un peu plus tard que les agriculteurs, les forestiers prennent très progressivement conscience qu'il n'y a plus de marchés captifs pour leurs produits et plus de consensus automatique sur les modes de gestion de leurs territoires et sur les aides afférentes. Cela ne veut pas dire que les forestiers ne détiennent pas des réponses potentielles intéressantes à des attentes fortes de la société, ou que la forêt soit inéluctablement appelée à redevenir un univers aux marges de la société, ni d'ailleurs que le bois ne dispose pas d'un important marché potentiel à conquérir ou reconquérir.

Mais cette situation nouvelle rappelle simplement qu'une politique forestière ne peut jamais être uniquement un "impératif catégorique" (clin d'œil à la morale selon Kant) dicté à la société par les acteurs privés et publics du secteur forestier qui sauraient mieux que quiconque ce qui est bon pour la forêt et pour la société. C'est en effet l'avènement du charbon qui a permis à la futaie de devenir la référence sylvicole dominante. C'est également l'amélioration des rendements agricoles et l'exode rural qui ont permis la réussite d'une politique d'extension forestière et d'accroissement de la ressource en bois. C'est bien le contexte économique des "Trente Glorieuses" qui a permis un long consensus sur l'augmentation quantitative des besoins français en bois, notamment d'industrie. C'est encore la fin des "Trente Glorieuses" qui a permis l'émergence d'un discours sur la priorité donnée aux bois de qualité. Les plus beaux textes juridiques et les plus belles techniques forestières ne sont pertinents et efficaces que dans la mesure où ils sont en harmonie avec les tendances lourdes de l'évolution d'une société. Les comportements sont très généralement en retard sur l'évolution socioéconomique et culturelle d'une société, mais ils finissent toujours par se modifier pour s'adapter à ces tendances lourdes, qu'il n'est pas toujours aisé de discerner sur le moment. Tôt ou tard les contradictions apparaissent, et lorsque l'adaptation a été trop lente, la "conversion" est trop souvent brutale et excessive.

Dans la situation encore confuse qui prévaut actuellement, il existe très schématiquement, parmi les parties prenantes au débat forestier, trois grands types de réaction. Un premier groupe prône la résistance à outrance à ce qui est perçu comme une "mode écologique" sans lendemain, un danger pour 


\section{Ch. BARTHOD}

la continuité indispensable à la réussite des investissements forestiers, et un désaveu pour tous ceux qui ont déployé une énergie énorme pour réussir une adaptation remarquable au contexte socio-économique et culturel des années 1950-80. Un second groupe rêve de l'opportunité offerte pour modeler la réalité forestière sur ce qui est largement un fantasme citadin, pense que la société a les moyens de prendre en charge les coûts certains qui sont afférents à ce rêve, et croit qu'il suffit de lois et de règlements pour imposer ce modèle normatif à ceux qui n'en veulent pas. Le troisième groupe croit que la politique forestière est un exercice d'équilibrisme qui se doit, d'une part de permettre l'émergence de solutions nouvelles adaptées aux tendances socio-économiques et culturelles lourdes de la société, sans en faire une norme, et d'autre part de vérifier à tout moment que ces solutions, issues d'idées et de connaissances nouvelles ou d'un compromis entre des tendances centrifuges, restent cohérentes avec la réalité forestière (ses contraintes théoriques, l'inertie des systèmes forestiers et le résultat escompté des efforts consentis précédemment) et n'engagent pas dans des impasses.

Seul ce troisième type de réaction peut à la fois combattre efficacement le second, et permettre une adaptation progressive et non traumatisante de la politique forestière française à ce nouveau contexte. II rappelle à ceux qui l'oublient que la vente de produits marchands demeure encore aujourd'hui la solution la plus performante pour financer une gestion durable. Ce n'est qu'en créant des conditions qui permettent aux produits forestiers de conquérir ou reconquérir des marchés rémunérateurs que le gestionnaire forestier pourra accepter de prendre en charge des contraintes environnementales supplémentaires. Le secteur aval doit en effet être en mesure de répercuter des coûts d'approvisionnement accrus lorsque les bois sont issus d'une gestion plus contraignante. Loin d'être une capitulation en rase campagne devant des "écologistes irresponsables", ou une conversion stupide à un irénisme économiquement intenable, cette stratégie lie fortement deux démarches paraflèles, en apparence contradictoires mais totalement complémentaires, II faut simultanément aller au devant des attentes de la société dans le domaine d'une prise en compte encore plus grande de l'environnement dans la gestion forestière (dans des limites raisonnables), et consentir un effort considérablement accru pour mieux insérer le secteur économique du bois et du papier dans le contexte technologique, financier et commercial moderne.

Si ces prémices sont acceptables, il est alors possible de rétléchir sur l'expérience des pays qui ont connu, et connaissent encore, un débat polémique beaucoup plus brutal que le nôtre, afin d'identifier certains défis qu'il est possible de chercher à relever ${ }^{(3)}$. Néanmoins, dans le contexte particulier de la forêt française, il sera alors également possible d'identifier clairement certaines questions difficiles, aujourd'hui sans réponse évidente, et dont la résolution est loin de dépendre de la seule initiative des forestiers. Le point de départ acceptable de cette double réflexion est le suivant: le forestier n'est pas seulement le gardien et l'éducateur d'une collection d'arbres. Sa responsabilité va bien au-delà. Elle concerne au premier chef le maintien de la structure et de la tertilite de sols en bon état de fonctionnement, la conservation de la diversité biologique des forêts à une échelle spatiale et temporelle qui doit être précisée, et la préservation d'écosystèmes forestiers dotés d'une bonne capacité de réaction aux perturbations inévitables, compte tenu de la longueur des cycles forestiers. Elle s'étend également à la satisfaction des besoins et attentes diverses de la société, dans le respect du droit de propriété inscrit dans la Constitution et borné par les lois.

\section{CINQ DÉFIS QUE LES FORESTIERS PEUVENT RELEVER}

- Le premier défi consiste à choisir clairement entre le renoncement au concept de foresterie multi-usages et une révision de la mise en œuvre du discours sur les trois fonctions (productrice, protectrice et sociale) de la forêt sur un même territoire. Ce qui est en cause est moins le

(3) La plus grande partie de la rédaction portant sur les cinq défis que les forestiers peuvent ralever est issue d'un article publie an 1995 dans le numéro 115 de la revue Aménagement at Nature, sous le titre "Le débat international sur la gestion das forêts". (Reproduit avec l'aimable autorisation de Aménagamant et Nature. - 21, rue de Collignon - 75116 PARIS). 
concept de foresterie multi-usages que la crédibilité de ses modalités pratiques de mise en œuvre. Une partie minoritaire mais influente de l'opinion publique pense parfois qu'il est possible de résumer la doctrine forestière traditionnelle par la maxime suivante: "ce qui est bon pour la production de bois réglée par un aménagement est nécessairement bon pour toutes les composantes de l'écosystème forestier ": c'est la théorie dite de l'effet de sillage. Dans beaucoup de cas, l'expérience séculaire d'une sylviculture raisonnable est loin d'infirmer toujours ce raccourci, néanmoins caricatural. Mais le courant contestataire n'a que faire d'une vision statistique de la réalité. Ses convictions sont fondées sur d'autres approches : d'une part sur des conflits locaux qui lui donne la référence de contre-exemples parfois criants, d'autre part sur une idée de nature qui n'est pas celle que s'est forgée collectivement le corps des gestionnaires forestiers.

Le talon d'Achille des forestiers réside dans la gestion des conflits inévitables entre certains usages dans des types de forêts particuliers. La question clé est souvent l'identification pertinente des enjeux majeurs et des projets des acteurs pour un territoire. Pour rendre crédible le discours de la foresterie multi-usages, il est indispensable que les forestiers perçoivent clairement les antagonismes entre usages tels qu'ils sont perçus par l'opinion publique, et acceptent d'arbitrer les conflits sans préjugé systématique en faveur des traitements sylvicoles traditionnels, même lorsque le rapport de force local n'est pas défavorable aux forestiers. Pour que le discours sur la foresterie multi-usages soit perçu comme cohérent, il faut que certains projets qui "coûtent" en matière de production de bois soient publiquement défendus par les forestiers, dans les régions où un antagonisme entre usages est fortement vécu par l'opinion.

C'est dire l'enjeu des forêts périurbaines, mais aussi de certains conflits locaux où la protection d'animaux emblématiques trouve une forte résonance dans les médias. Pour le public, la charge de la preuve appartient en grande partie aux forêts publiques, car la taille des propriétés privées pose le problème différemment et l'opinion reste toujours globalement attachée au respect du droit de propriété. Si les forestiers publics ne relèvent pas ce défi, il est à craindre qu'on ne s'achemine rapidement vers un zonage officiel du territoire forestier en fonction des vocations principales, avec définition d'une procédure d'arbitrage des conflits entre usages. Cette solution est réclamée par ceux qui ne font plus confiance aux forestiers dans leur mise en œuvre du concept de foresterie multiusages, pourtant au cœur des discours forestiers depuis près d'un demi-siècle. Même sans vouloir argumenter en détail sur l'opportunité politique très contestable d'une telle solution, elle est parfaitement irréaliste en France, compte tenu de la structure du foncier forestier français, de la répartition de l'habitat rural, et de l'ancienneté de l'empreinte humaine sur la forêt, qui fait coexister des peuplements très anthropisés (taillis, et mélanges de taillis-sous-futaies) et des peuplements davantage susceptibles de s'intégrer dans un tel choix.

- Le second défi consiste à revoir la stratégie générale de conservation en forêt et mieux intégrer la conservation de la biodiversité dans la gestion forestière ordinaire. Indèpendamment de ses fondements éthiques et scientifiques, la demande est certainement liée à la nouvelle perception de la nature par une société majoritairement citadine, à la recherche de son enracinement dans un terroir et dans l'immuabilité. Comme il existe une passion pour les vieilles pierres, garantes de notre histoire et de notre origine, il existe désormais une fascination pour une forêt éternelle, prèservée des errements de l'action humaine (4). Si ce mouvement est parti d'Amérique du Nord, c'est que la beauté grandiose et fascinante des forêts anciennes de la côte Nord-Ouest du Pacifique frappe tous ceux qui les parcourent. L'enjeu en est la préservation intégrale de certaines forêts très peu touchées par l'homme ${ }^{(5)}$, ou la recréation, sur des surfaces à définir, d'une dynamique forestière sans inter-

(4) MIDDLETON (D.). - Ancient Forests : a celebration of North America's old-growth wilderness. - 1992. - $107 \mathrm{p}$.

(5) NORSE (E.A.). - Ancient forests of the Pacific Northwest: the grandeur, complexity, diversity and impending destruction of a fragile and vital ecosystem. - Washington (DC) : Island Press, 1990. - $327 p$.

ALVERSON (W.S.), KULHMANN (N.), WALLER (D.M.). - Wild Forests : conservation biology and public policy. - Washington (DC)

Island Press, 1994. - $300 \mathrm{p}$ 


\section{Ch. BARTHOD}

férence avec l'homme. Dans le cadre d'une des résolutions de la Conférence d'Helsinki, la France s'est engagée à créer un réseau de forêts anciennes et subnaturelles, et l'Office national des Forêts (ONF) travaille très activement à sa mise en œuvre, avec la fin de l'année 1996 comme échéance pour la publication d'une instruction technique. Même si la motivation essentielle en est l'intérêt scientifique pour l'étude des processus d'évolution des écosystèmes forestiers, la réponse à une demande symbolique forte de la société mérite également considération.

Mais, dans tous les pays de l'hémisphère Nord, on constate une certaine réticence des forestiers à retirer de la gestion des surfaces significatives, car ce type de décision est souvent vécu comme un échec et une contestation du travail "civilisateur" du forestier. Jusqu'à présent, dans pratiquement tous les cas, la décision de mettre en réserve intégrale certains territoires forestiers d'une taille significative a été le fruit d'un processus complexe de négociation-confrontation avec des ONG relayées par les médias. II est encore trop tôt pour savoir si la mystique de la "wilderness" (6), si prégnante en Amérique du Nord (7), avec son cortège de considérations sur la régénération morale de l'homme au contact avec une nature pratiquement intouchée par l'homme, s'imposera durablement en Europe. Tout au plus peut-on souligner qu'il en existe certaines prémices, notamment dans la culture germanique et scandinave.

Un autre aspect de ce défi est la place qui sera faite aux autres composantes de l'écosystème forestier que les arbres des essences principales, dans la gestion forestière ordinaire. Pour la culture forestière européenne, il s'agit d'une évolution sensible par rapport aux préoccupations dominantes lors des cinquante dernières années, mais pas d'une révolution culturelle. L'esprit naturaliste et la curiosité pour toutes les formes de vie et d'associations végétales et animales, qui ont caractérisé la foresterie française de la fin du XIX et du début du XX $X^{e}$ siècles, sont encore vivants et ne demandent qu'à se ranimer. Dès que la question de la conservation des ressources génètiques forestières s'est posée en termes opérationnels, un groupe de travail national a été créé en 1986 sur ce sujet, parallèlement à un groupe de travail sur la conservation des milieux représentatifs, remarquables ou en danger. Après une réflexion sérieuse, le ministère chargé des forêts a publié en 1991 une circulaire sur la conservation des ressources génétiques forestières, et en 1993 une circulaire sur la prise en compte de la biodiversité dans la gestion forestière. Dans le droit fil de cette rétlexion, l'ONF, qui accueille par ailleurs dans les forêts domaniales l'essentiel du programme français de conservation des ressources génétiques forestières, a élaboré une remarquable instruction sur la biodiversité en forêt, qui représente une option pertinente et courageuse, intègrée dans une vision cohérente des enjeux majeurs de la conservation de la biodiversité en forêt. Ces documents d'orientation s'insèrent dans le courant européen qui s'est exprimé dans la résolution $\mathrm{H} 2$ de la Conférence d'Helsinki, sur la conservation de la diversité biologique dans les forêts européennes.

Si un corps de doctrine français semble en bonne voie de définition, le défi majeur réside dans l'intégration pratique de cette préoccupation renouvelée pour la conservation de la biodiversité au niveau du terrain, c'est-à-dire dans les compromis qu'il faut inventer au jour le jour entre des logiques qui ne sont pas toujours convergentes. Si la question des mesures de protection de certaines espèces et de certains milieux par le recours à des réserves intégrales n'est déjà pas toujours facile à résoudre, celle de l'élaboration du cahier des charges de gestion de réserves dirigées est encore plus complexe, sans parler des choix sylvicoles dans le reste du territoire forestier. La conception et

(6) Concept nord-américain célébrant la nature sauvage et grandiose, sans marque de l'homme; ce concept a été notamment illustré par Henry David Thoreau (1817-1862 : pacifiste influence par la mystique hindaue et l'idealisme allemand, theoricien de la non-violence et de la désobéissance civile, écrivain populaire célébrant la nature et les bienfaits qu'en retirent ceux qui vivent à son contact immédiat), John Muir (1838-1914: découvreur des beautés de la nature sauvage dans la chaine de la Sierra. promoteur de l'idée des grands parcs nationaux pour préserver intégralement des régions encore inaccessibles, á l'origine du premier parc national californien (Yosemite) et père fondateur du Sierra Club qui compte aujourd'hui plus de 400000 cotisants) et Aldo Leopold (1887-1948: forestier américain et essayiste, assistant-directeur pour les forêts fédérales de l'Arizona et du Nouveau Mexique, puis directeur adjoint de I'US-Forest Products Laboratory enfin professeur de gestion de la faune et de la flore sauvages à l'Université du Wisconsin, premier défenseur américain d'une approche écosystemique de la forêt, avocat d'une réhabilitation du rôle des animaux dits nuisibles, fondateur de la Wilderness Saciety).

(7) OELCHLAEGER (M.). - The idea of wilderness. - New-Haven, London : Yale University Press, $1991 .-477 \mathrm{p}$ 
la mise en ceuvre des documents d'objectifs de gestion des forêts du réseau européen Natura $2000^{\left({ }^{(8)}\right.}$, sous l'ègide du ministère de l'Environnement, le montreront sans nul doute. La gestion suppose des choix et implique très généralement une certaine simplification locale du milieu. Encore faut-il s'apercevoir à temps du moment où l'on franchit la ligne jaune, pour pouvoir corriger la direction empruntée. C'est l'enjeu du débat encore balbutiant sur les inventaires préalables aux décisions majeures et sur les espèces indicatrices, réputées révéler précocement une réorientation, positive ou négative, du fonctionnement de l'écosystème forestier. Un dernier élément de ce second défi consiste à rendre visible celte préoccupation de biodiversité, et à élaborer des procédures d'évaluation des actions menées dans ce sens, dans le fonctionnement ordinaire d'un service de gestion forestière. En effet, les meilleures circulaires ou instructions ne sont pas en elles-mêmes des gages suffisants, si l'évaluation des organisations et des agents ne prend en compte que des paramètres économiques facilement mesurables.

- Le troisième défi consiste à diversifier les options sylvicoles et à réexaminer le quasi-monopole de fait dont jouit, dans les orientations de gestion et dans les aides, le traitement en futaie monospécifique régulière équienne. La demande porte sur la diversité en forêt, diversitè visible au travers des essences et des traitements sylvicoles, et diversité invisible (pour la plupart des promeneurs) au travers des espèces animales ou végétales souvent discrètes. Sans que soit nié le besoin économique de peuplements forestiers à forte productivité, gérés très intensivement, que le public n'identifie pas vraiment avec l'image mythique de la forêt, il existe un rejet grandissant à l'encontre de toutes les pratiques sylvicoles qui homogénéisent la forêt sur de vastes étendues, là où il pourrait exister de la variété. Un présupposé de cette perception est que la nature fabrique de la variété et que l'action de l'homme est nécessairement réductrice de cette variété, ce qui est loin d'être partout vérifié, comme le montrent certaines études sur la dynamique naturelle des écosystèmes forestiers. La comparaison de Pinchot, illustre forestier américain du début du siècle, assimilant la sylviculture avec la culture d'un champ de maïs, est désormais inacceptable pour nos sociétès citadines modernes. Ce qui symbolise le mieux ce rejet est la plantation monospécifique de résineux exotiques.

D'une certaine manière, cette évolution invite les forestiers français à redécouvrir les origines et la richesse de leurs traditions sylvicoles, parfois déformées par le développement de certaines pratiques sylvicoles qui ne datent que de quelques décennies, au maximum du début du siècle (purification spécifique de certaines futaies, option pour la plantation aux dépens de l'enrichissement progressif des peuplements preexistants, introduction de certaines espèces hautement productives dans des milieux qui ne leur conviennent pas nécessairement, etc...). Ceci ne doit pas faire oublier que ces choix n'ont affecté qu'une proportion relativement réduite de nos forêts, même si leurs effets sont parfois très visibles sur le paysage; il suffit de comparer la situation française avec celle des pays de tradition germanique. Par ailleurs certains de ces choix trouvaient partiellement leur origine dans la réaction contre les errements de certaines pratiques antérieures qui n'avaient pas que des avantages. La grande tradition forestière française de la futaie rẻgulière mélangée, renouvelée par régénération naturelle, ne suffira sans doute pas à résoudre toutes les questions d'aujourd'hui, mais elle peut être source d'inspiration, sans imitation servile. II existe une place en France pour une pluralité de modèles syłvicoles, et rien n'impose de rechercher à privilégier les mêmes options quels que soient le contexte écologique et social, les objectifs et les contraintes du propriétaire. Il faut savoir revenir aux cahiers des charges auxquels les modèles sylvicoles prétendent répondre, pour éviter de transformer des interrogations légitimes sur les limites inhérentes à chaque sylviculture en "guerre de religion".

La foresterie française a toujours été pauvre, comparée avec celle de la plupart de nos voisins; elle a même été regardée avec condescendance par d'autres écoles sylvicoles dotées de moyens plus importants pour artificialiser le milieu forestier. C'est sans doute une des raisons majeures pour les-

(8) Rèseau communautaire, en cours de création en application de la directive européenne de 1992 sur la protection des habitats, de la faune et de la flore. 


\section{Ch. BARTHOD}

quelles elle a "choisi" d'imiter au mieux la nature, selon les termes mêmes de la célèbre maxime du forestier français Parade, au XIXe siècle: "imiter la nature, hâter son œuvre ". D'une certaine façon, cette pauvreté est devenue vertu. Le contexte des cinquante dernières années avait un peu bouleversé la donne. Or le renchérissement extraordinairement rapide des travaux sylvicoles met en quasifaillite certains modèles sylvicoles trop vite devenus des références quasi obligées, notamment en forêt privée. L'investissement initial lors d'une plantation sur de vastes surfaces devient prohibitif pour beaucoup de propriétaires privés, quel que soit le résultat théorique des calculs micro-économiques d'actualisation, sauf peut-être dans le cas de modèles de ligniculture. Cette situation conduit à s'intéresser à nouveau à des modèles sylvicoles plus économes d'intrants, travaillant sur les peuplements préexistants, et à redécouvrir les mélanges taillis-futaie, encore si répandus dans les forêts privées. Au-delà de certaines querelles dogmatiques sans grand intérèt, c'est l'enjeu essentiel du débat sylvicole relancé par l'association Pro Silva. Un peu partout en Europe et en Amérique du Nord (mais encore peu en France), la recherche essaie d'apporter aux sylviculteurs une contribution à cette réflexion, à partir de l'étude de la dynamique des écosystèmes forestiers subnaturels et des perturbations naturelles. Mais le débat court sans cesse le risque de déraper dans un affrontement idéologique qui ne dirait pas son nom, sur l'idée de nature. C'est un des problèmes difficiles auxquels sont confrontés actuellement les sylviculteurs.

- Le quatrième défi consiste à revoir certaines des options actuelles en matière d'aménagement du territoire forestier. Une des perceptions les plus fortes du milieu forestier est parfois celle des infrastructures et des grandes options d'aménagement du territoire forestier, notamment à l'échelle du paysage lorsque le relief le permet. Dans certains cas, elle heurte profondément l'idée de nature que se fait le citadin, car elle imprime nettement la marque de l'homme et du contingent dans un milieu dont il voudrait croire qu'il n'est que nature et immuabilité. C'est pourquoi une sensibilité nouvelle de l'opinion publique porte sur le paysage, sur la taille et la géométrie des parcelles, sur les routes forestières, sur les coupes rases, sur le traitement des lisières, etc... Les forestiers ont souvent hésité entre deux attitudes. La première consiste à vouloir rappeler le citadin à la réalité des pratiques forestières, considérées comme intrinsèquement bonnes pour la forêt, à ne rien cacher des phases ingrates que peut connaître un cycle forestier et à tenter de persuader le public de la validité des choix sylvicoles ou d'aménagement, en faisant confiance au temps et à la nature pour panser des plaies aujourd'hui très visibles. La seconde consiste à ne sacrifier que ce qui est strictement nécessaire pour éviter la contestation, en prévoyant un traitement esthétique limité à ce qui est perçu par le public dont on sait qu'il entre généralement peu profondément en forêt. Mais la réponse n'est pas toujours à la hauteur de la question. Faut-il vraiment chercher à généraliser dans tous les types de milieux des options techniques lourdes qui ont surtout fait leurs preuves dans des milieux à fortes potentialités productives? Ne doit-on pas accentuer encore le mouvement actuel de différenciation des intensités de gestion selon les types de milieux et de paysages?

Parmi les fondements ancestraux de l'éthique forestière, hérités du contexte des huit derniers siècles, se trouve le devoir de prévenir une éventuelle "faim de bois" de la nation, en développant sans cesse la ressource et en intensifiant la production et la récolte de bois partout où cela est possible. Au cours des cinquante dernières années, grâce à des moyens techniques et financiers nouveaux, des travaux considérables ont été ainsi menés sur des pentes importantes ou sur des sols difficiles, nécessitant des travaux d'infrastructure non négligeables, souvent marquants dans le paysage. La rentabilité micro-économique n'en est pas toujours très évidente, et l'intérêt pour la nation peut quelques fois en être discuté. Même s'il est indispensable d'être cohérent sur le moyen terme dans les efforts de boisement et reboisement, en tenant compte des nécessaires équilibres de la ressource en classes d'âge, en diamètres et en qualités des bois, le devoir de prévenir une "faim de bois" ne peut plus être le fondement majeur des choix de la politique forestière.

Par ailleurs, la fermeture récente de certains paysages à la suite de boisements importants suscite parfois un malaise des populations locales, en l'absence d'une vision claire sur le devenir de certains terroirs. Le paysage est certes un concept particulièrement difficile à manier dans un cadre adminis- 
tratif, et il est à craindre que ne s'impose un "bon goût" officiel, conservateur en diable ou élitiste, génèrateur d'arbitraire pour les citoyens. Mais la contestation actuelle révèle sans aucun doute un malaise qui mérite réflexion. Se pose alors rapidement, dans un pays marqué par une structure relativement morcelée de la propriété, la question de l'opportunité et de la légitimité de contraintes nouvelles imposėes aux propriétaires fonciers au nom de références esthétiques difficiles à cerner, dans le cadre de procédures qui risquent de connaître des dèrives bureaucratiques redoutables.

La sensibilité nouvelle au paysage, à la géométrie et à la taille des parcelles forestières, aux impacts visuels des routes forestières, à la taille des coupes rases, ou au traitement des lisières nécessite incontestablement que les forestiers s'en préoccupent. La meilleure solution serait qu'ils prennent par eux-mêmes l'initiative d'élaborer une sorte de déontologie professionnelle, faute de quoi des pressions indistinctes risquent d'aboutir à èdicter une réglementation nouvelle, lourde d'application et difficile à moduler en fonction des cas de figure locaux. Certaines initiatives de l'ONF vont incontestablement dans le bon sens, mais la question la plus difficile à résoudre dècoule de l'interférence involontaire d'une série de décisions individuelles des propriétaires fonciers privés. On voit déjà des parcs naturels régionaux tenter d'interdire le financement par l'État de certaines techniques forestières et certaines essences, sans négociation avec les reprèsentants des propriétaires fonciers, ce qui est une voie dangereuse et démocratiquement malsaine. Ceci rèvèle à l'évidence qu'un problème existe, mais qu'on ne sait actuellement pas vraiment comment le traiter.

- Le cinquième et le plus lourd défi consiste à réexaminer les procédures d'information, de consultation et d'association du public sur les choix majeurs. Les forestiers ont une conscience aiguë de travailler dans un domaine sans équivalent, de par les durées mises en jeu par chaque décision, où les raisonnements par analogie à partir d'autres secteurs d'activité peuvent être stimulants, mais jamais entièrement convaincants. C'est la raison pour laquelle ils oscillent souvent entre deux attitudes que leurs interlocuteurs ne considèrent gẻnéralement pas comme pertinentes, mais qui sont aisément compréhensibles et parfois justifiées. La première consiste à tenter de persuader l'opinion publique du bien-fondé de leurs analyses et décisions, présentées comme la seule voie raisonnable possible ; la communication est alors essentiellement conçue comme "propagande", ne retenant des questions qui sont posées aux forestiers que celles dont les rèponses légitiment la politique forestière menée ou minimisent les inflexions inévitables. La seconde consiste à éviter au maximum les interfėrences provenant de l'extérieur avec leurs procédures internes de décision, de façon à écarter les choix trop enracinès dans une conjoncture précise et probablement fugitive, dans les convictions particulières d'un petit nombre d'individus, dans les effets de mode ou dans des stratégies politiques à court terme. Cette seconde attitude conduit aussi souvent à se méfier des pressions locales et donc à encadrer, parfois excessivement, la nècessaire marge de liberté des échelons d'exécution.

La première attitude se heurte de plus en plus à la réaction de ceux qui, de plus en plus nombreux. connaissent bien tel ou tel aspect de la réalité forestière, ainsi qu'à la mention publique de contreexemples difficiles à gérer dans un contexte de "propagande". La seconde est de plus en plus mal vécue dans des sociétés démocratiques où la participation et la transparence dans les processus de prise de décision sont vécues comme un droit et une garantie que les opinions contradictoires seront effectivement entendues. La première et la seconde sont intenables à terme quand le monopole administratif et technique des forestiers sur la forêt est rompu, provoquant une série de négociations séparées avec tel ou tel groupe d'intèrêt qui sait souvent mieux que les forestiers diffuser et faire partager ses informations et ses convictions. C'est dire qu'on se retrouve insidieusement dans la pire des situations pour la logique des dẻcisions forestières, celle des groupes de pression "naturels" ou autoproclamés, agissant sur des segments atomisès des choix d'aménagement et de sylviculture. II faut sortir rapidement de cette impasse qui ne satisfait personne.

II s'agit d'un des enjeux majeurs de la relance des commissions régionales de la forêt et des produits forestiers (CRFPF), décidée en 1995 et accompagnée d'une proposition de réflexion régionale sur la gestion durable des forêts. Il appartient aux partenaires forestiers de relever ce dèfi d'un dialogue, même difficile, voire conflictuel, mais organisé au sein des CRFPF, avec l'ensemble des orga- 
nismes et associations qui revendiquent d'être écoutés préalablement à la définition des grandes options de politique forestière et à la résolution des conflits locaux. Mais ceci ne suffira sans doute pas à satisfaire une demande croissante d'information, de consultation et d'association du public sur les choix majeurs. D'autres mécanismes et procédures devront être imaginés. Par exemple, dans l'objectif de mieux prendre en compte les demandes des populations locales vis-à-vis des grandes orientations de gestion des forêts publiques, peut-être serait-il souhaitable de consulter systématiquement les conseils municipaux des communes sur lesquelles se trouvent des forêts domaniales, ainsi qu'un certain nombre des partenaires ordinaires de l'ONF, dans le cadre de procédures préalables à l'élaboration des aménagements.

Trouver une réponse crédible à l'ensemble de ce cinquième défi suppose, d'une certaine façon, de sortir d'une logique de mono-appropriation d'un espace forestier que se disputent différents intérêts ou usages qui cherchent à s'exclure ${ }^{(9)}$. Actuellement, en apparence, nul n'a intérêt à ce que la qualité biologique totale des forêts se dégrade mais, du point de vue d'une analyse système-acteurs, la proposition doit malheureusement être renversée: nul, sinon le propriétaire foncier (qui en assume la plus grande partie de la charge financière), n'a vraiment intérêt à ce que la qualité biologique totale de la forêt ne se dégrade. Dans ce contexte, toute approche purement "techniciste" de la gestion durable est radicalement insuffisante si le forestier n'accepte pas d'entrer dans une démarche de gestion patrimoniale des forêts, car il est loin de pouvoir maîtriser à lui seul l'ensemble des paramètres qui interfèrent avec ses propres choix et objectifs. Cela suppose une identification de tous les acteurs, qui sont parfois indifférents mais plus souvent inconscients de l'impact de leurs décisions sur la forêt, et dont le comportement quotidien pèse lourdement sur l'avenir de massifs forestiers proches ou lointains du lieu de leurs activités. II faut donc que les forestiers sortent du milieu forestier et travaillent à créer les conditions qui permettent à tous ces acteurs de voir dans la forêt un patrimoine commun, de lui donner une place dans le champ des intérêts qu'ils prennent en charge, dont ils se sentent responsables, et que cette prise en charge soit acceptable pour eux par rapport au comportement de l'ensemble des autres acteurs concernés. Sans cette révolution culturelle, il n'y aura pas de gestion durable des forêts travaillées par l'homme.

\section{LES QUESTIONS ENCORE TROUBLES DU DÉBAT SUR LA GESTION DURABLE DES FORÊTS}

- Une des originalités fortes de la forêt française au niveau international réside dans l'étendue des taillis et des mélanges de taillis et de futaies, hérités du traitement historique en taillissous-futaie. À côté de 6,8 millions d'hectares de futaies régulières ou irrégulières, il existe encore 6,9 millions d'hectares de tels types de peuplements, plus d'un siècle après l'affichage d'une forte option politique en faveur du traitement en futaie. Ces 6,9 millions d'hectares sont l'héritage de modes de traitements totalement artificialisés, correspondant à un mode ingénieux d'exploitation des potentialités des ligneux pour répondre à des besoins très précis et diversifiés. Même si la revalorisation du bois de feu redonne une certaine valeur à ces formations, leur avenir demeure une des préoccupations majeures de la politique forestière française. Les modèles sylvicoles prônés par les différents courants de pensée qui réfléchissent aux conditions d'une gestion durable correspondent tous à un traitement en futaie.

Certes, à l'échelle des millénaires et de la science écologique, la végétation forestière saura évoluer, et la dégénérescence actuelle ou prévisible des taillis inexploités ou non renouvelés, jointe à l'absence de jeunes tiges de futaie, ne devrait pas conduire en fin de compte à une végétation steppique. Certes, la forêt n'a pas besoin de l'homme pour subsister, comme le font remarquer à très juste titre les maîtres à penser de nos écologistes, mais cette ironie facile oublie un peu vite la res-

(9) BAATHOD (Ch.), OLLAGNON (H.). - Vers une gestion patrimoniale de la protection et de la qualite biologique des forêts. - Revue forestière française, vol. XLV, $n^{\circ} 2,1993, p p .159-163$. 


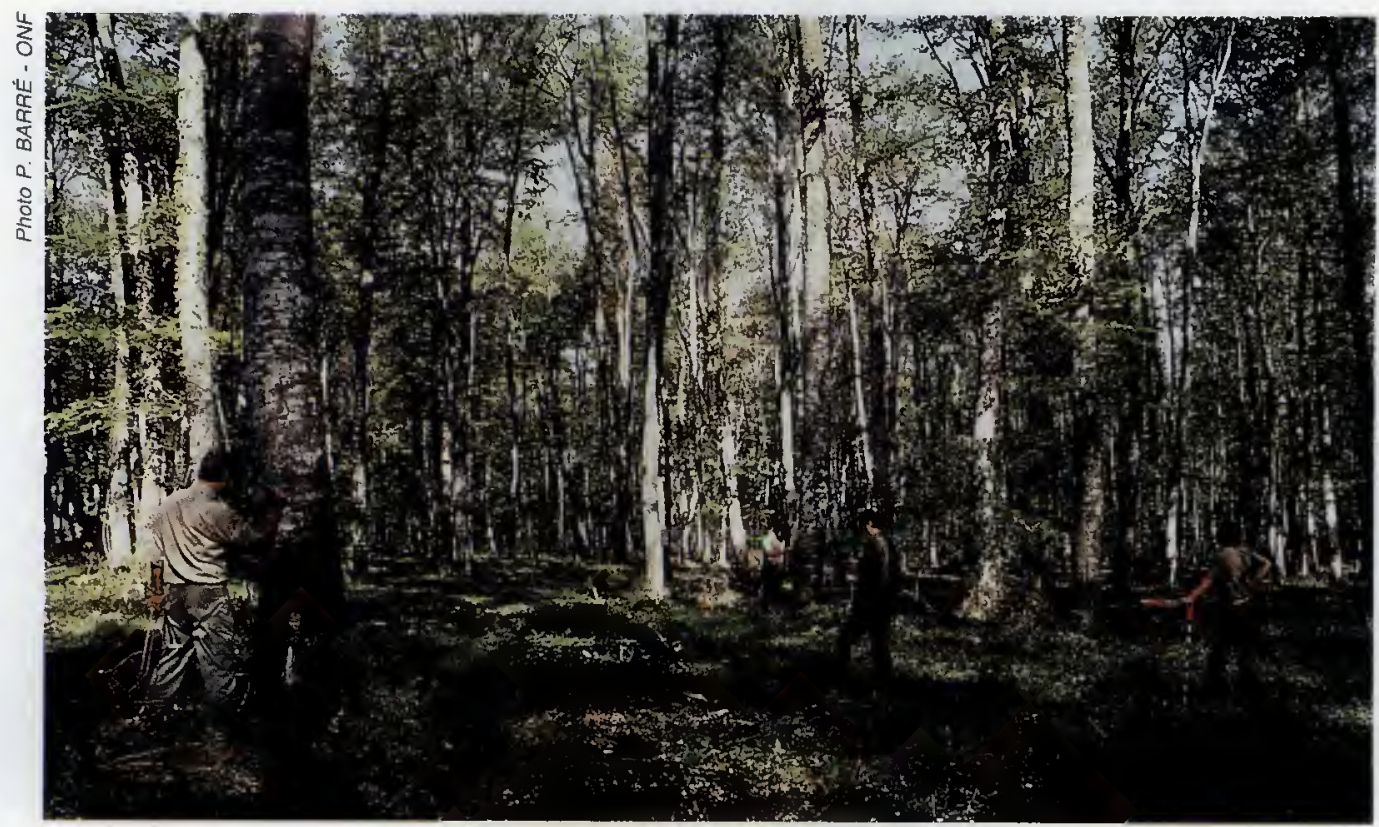

ponsabilité de la politique forestière vis-à-vis de la réorientation des formations forestières très anthropisées qui n'ont pas d'équilibre en dehors de l'action de l'homme. II est d'ailleurs amusant et un peu paradoxal que certains milieux écologistes attaquent à la fois les forestiers pour avoir cautionné l'extension de systèmes biologiques simplifiés et dépendants de l'homme, dans l'objectif de répondre à des demandes pressantes de la société, et soulignent avec force la contribution de ces formations de taillis et mélanges de taillis et de futaies à la conservation de la biodiversité en forêt, en fournissant des habitats intéressants.

Si on écoute certains discours un peu caricaturaux, les taillis, les taillis-sous-futaie et les futaies irrégulières sont à protéger, le grand ennemi diabolisé étant la futaie régulière (10), quelles que soient ses modalités d'application (taille des parcelles, mélange ou non des essences, présence ou non d'un sous-bois, régénération naturelle ou non,...). En toute rigueur, l'assimilation de la futaie régulière à de la forêt relevant de "l'imposture" et de "l'inquiétant mariage du forestier et de l'agronome", la forêt française semble donc se réduire au 0,74 million d'hectares de futaies irrégulières, selon certains points de vue de militants écologistes. Le traitement en futaie jardinée, à tout le moins en futaie irrégulière mélangée, est alors promu à l'état de seul traitement compatible avec une gestion durable. C'est dire la marge qui existe entre les discours des théoriciens scientifiques de la gestion durable et ce qui en est récupéré dans les combats militants. La futaie régulière semblant récusée une fois pour toutes par beaucoup de nos partenaires écologistes, et toute discussion pragmatique étant excessivement difficile sur ce sujet avec les militants de la futaie jardinée, quel avenir est-il possible de dessiner pour les 6,9 millions d'hectares de taillis et de mélanges de taillis et de futaies, les modalités de conversion traditionnellement prônées par l'ONF ne semblant pas en mesure de répondre à l'ampleur de ce défi. Les forestiers restent donc seuls pour tenter de trouver une réponse pragmatique, techniquement et financièrement acceptable pour le propriétaire.

(10) CARBIENER (D.). - Les Arbres qui cachent la forêt : la gestion forestière à l'épreuve de l'écologie. — Aix-en-Provence : Édisud, 1995. - 243 p. (publiẻ avec le soutien du WWF). 


\section{Ch. BARTHOD}

- La seconde des grandes originalités françaises au niveau international réside dans la très forte proportion des forêts privées et l'absence quasi totale d'intégration économico-juridique entre la forêt et l'industrie du bois. Alors qu'en moyenne la forêt privée n'appartenant pas à des groupes industriels ne représente qu'entre le tiers et la moitié de la forêt des pays d'Europe occidentale ou nordique, elle représente près des trois quarts en France, avec environ 3,7 millions de propriétaires forestiers, dont seule une infime minorité tire de la sylviculture le principal de ses revenus. La structure foncière forestière française rend à elle-seule illusoire toute stratégie de zonage réservant à une dynamique naturelle de végétation de vastes zones compatibles avec la conservation de la diversité biologique, et laissant le champ libre à une sylviculture de plus en plus inspirée de l'agriculture sur le reste des territoires. La politique forestière française est condamnée à rechercher une approche intégrée de la gestion durable sur tout le territoire forestier, sans exclure bien sûr des modulations dans les objectifs privilégiés localement. En France, sans qu'il soit par ailleurs possible d'oublier que les forêts domaniales appartiennent au domaine privé de l'État, le débat sur la gestion durable des forêts ne peut donc pas éviter de devenir aussi un débat sur la conception du droit de propriété, d'autant plus qu'une grande majorité des écologistes ne sont pas propriétaires fonciers en milieu rural.

La forêt est notamment vécue par beaucoup de propriétaires forestiers comme le lieu d'une liberté et d'une responsabilité garanties par le droit de propriété, dans le respect des réglementations qui sont seules à pouvoir incarner la charge de l'intérêt général et les demandes légitimes de la société. La forêt est alors le lieu d'une mémoire, un héritage reçu et à transmettre après avoir fait produire un intérêt, un lien avec l'avenir et la garantie d'une solidarité paisible entre les générations. Avant même d'être un bien économique, la forêt est un patrimoine, revêtu d'une forte valeur symbolique et affective, et suscitant des attitudes particulières. L'adoption à un rythme rapide de nouvelles réglementations environnementales, avant même qu'un équilibre ait été trouvé pour les modalités d'application des textes précédents, conduit à une déstabilisation permanente des propriétaires qui se sentent alors confrontés à un arbitraire administratif. Par ailleurs, en l'absence d'une réflexion sur le contenu d'une gestion normale, il est excessivement difficile de poser correctement les termes d'une éventuelle compensation financière (ou indemnisation) du propriétaire forestier, lorsque la société impose les contraintes d'une gestion particulière sur une partie du territoire boisé, au nom d'un intérêt supérieur (protection d'une espèce en danger, par exemple).

Dès lors les objectifs légitimes de la gestion durable et de la conservation de la diversité biologique apparaissent en partie comme concourant à une remise en cause de l'exercice du droit de propriété, d'autant plus que ces contraintes sont présentées comme non discutables car fondées sur une approche scientifique, et qu'il n'existe pas de procédure de recours gracieux, avant un recours éventuel au tribunal administratif. Alors même que la plupart des propriétaires forestiers sont assez sensibles à la nécessité d'infléchir certaines modalités de gestion el à leur responsabilité vis-à-vis de l'environnement, la solution de la formation, de la vulgarisation et de la négociation ne semble pas retenir assez l'attention des décideurs. II est parfaitement mensonger de réduire le propriétaire forestier à un "homo oeconomicus" borné et fermé à toutes les préoccupations d'un honnête homme et d'un citoyen, tout comme il serait absurde de penser pouvoir exiger de lui des investissements importants sans aucun espoir de rentrer dans ses fonds, à tout le moins d'en retirer les satisfactions qu'il estime devoir découler du temps et de l'argent immobilisés. II existe donc un devoir de formation, de vulgarisation et de négociation, certes difficile à mettre en œuvre, mais sur lequel il faut également parier. En le refusant, il se met en place insidieusement un contexte favorable à un débat confus et malsain mêlant inextricablement gestion durable et remise en cause du droit de propriété, risquant ainsi de pénaliser lourdement une politique de promotion d'une plus grande gestion durable.

- Du point de vue des références administratives et réglementaires, la forêt se trouve actuellement coincée entre deux modèles, celui de l'agriculture et celui du foncier urbain. Dans le secteur agricole, très fortement subventionné, les orientations politiques font l'objet d'une cogestion de fait entre les pouvoirs publics et les organisations professionnelles agricoles, sur la base d'un pro- 
cessus quasi permanent de négociation. Les dispositifs incitatifs sont très nettement privilégiés par rapport aux contraintes réglementaires, et ces dernières sont toujours accompagnées d'une riche palette d'aides financières permanentes ou temporaires. Le monde forestier aspire majoritairement à bénéficier d'un traitement comparable, en oubliant parfois que ce modèle s'accompagne de très fortes contraintes sur les orientations de production (cf. les quotas laitiers, par exemple).

II est pourtant évident que la société française et l'Union européenne ne consentiront jamais au profit des forestiers un effort comparable à celui qu'elles acceptent pour les agriculteurs, au seul motif de soutenir leurs revenus. De plus, les pays nordiques ne pourront jamais accepter la mise en place d'un dispositif cohérent d'aides qui minore l'avantage commercial comparatif dont ils disposent actuellement. Par ailleurs, des pays comme l'Allemagne et le Royaume-Uni sont politiquement très hostiles à toute intervention communautaire ou étatique dans le secteur forestier, au nom d'une certaine conception du libéralisme économique et de la subsidiarité entre les niveaux communautaire et national. Enfin, le monde agricole, actuellement soumis à des difficultés énormes d'adaptation à une nouvelle conjoncture, n'est actuellement pas prêt à accepter ce qui passerait inévitablement par un redéploiement budgétaire en faveur de la forêt. Ce n'est donc vraisemblablement pas le modèle agricole qui servira de référence pour dessiner l'essentiel du cadre administratif et réglementaire d'une promotion de la gestion durable des forêts. II n'est cependant pas inutile de rappeler que ce modèle négocié est assez largement à la base de la gestion environnementale de certains pays comme le Danemark, les Pays-Bas, ou l'Autriche, au moins en parallèle à des approches purement réglementaires.

Le seul modèle alternatif proposé aux forestiers est directement inspiré de la gestion du foncier urbain. Ce modèle repose sur des zonages correspondant à des niveaux variables de contraintes, faisant appel à des commissions administratives et parfois à des enquêtes publiques, sans que le propriétaire puisse contrôler la réévaluation ou la dévaluation financière de fait de son bien qui en découlent, ni même prétendre à des mesures compensatoires. C'est cette option qui est plus ou moins à la base du corpus législatif et réglementaire qui se met en place en matière de droit de l'environnement. Elle a l'énorme avantage de permettre un volontarisme administratif sans impliquer de lourdes dépenses publiques. Les enjeux financiers du foncier urbain justifient et atténuent un peu l'ampleur des contraintes réglementaires et son cortège inévitable d'arbitrages ressentis comme arbitraires par ceux qui en subissent les contrecoups. Dans le domaine forestier, le sentiment d'arbitraire reste brut et devrait largement contribuer à alimenter une certaine démotivation des propriétaires. Cette stratégie découle directement de la faiblesse des moyens humains et tinanciers du ministère de l'Environnement, que les forestiers ne peuvent que déplorer.

Dans ce contexte, une interrogation majeure porte sur l'opportunité éventuelle que le ministère en charge des forêts prenne l'initiative de répondre aux attentes de l'opinion publique et d'anticiper ainsi (avec une sensibilité un peu différente) une partie des orientations du ministère de l'Environnement, très semblables par ailleurs à ce qui se met en place dans les pays germaniques, anglo-saxons et nordiques. Un tel choix conduirait alors à promouvoir des approches contractuelles dans le domaine de la promotion d'une plus grande gestion durable et de la conservation de la diversité biologique en forêt. Faudrait-il aller pour cela jusqu'à redéployer certaines aides actuelles, ef conditionner davantage les subventions au boisement ou reboisement, voire certaines dispositions fiscales, comme sont en train de le faire certains de nos partenaires européens? Les propriétaires forestiers sylviculteurs seraient-ils désireux de s'engager dans une telle approche, qui semble aujourd'hui gagner du terrain dans beaucoup de pays occidentaux et être seule à même d'éviter l'amoncellement des réglementations contraignantes? Le paysage français est rien moins que clair sur ces questions.

- Fondamentalement la foresterie est à la fois une science et un "art". On oppose généralement le scientifique qui doute ef l'ingénieur qui saif. Ceci n'est pas entièrement faux, ef trop souvent les forestiers donnent l'impression d'avoir une réponse définitive à chacun des problèmes que soulèvent leurs partenaires. L'évolution des questions et des connaissances ne peut pourtant inspirer aux praticiens qu'une certaine humilité, même s'ils n'ont pas du tout à rougir de leurs actions et du résultat 


\section{Ch. BARTHOD}

des actions de leurs prédécesseurs. Mais la différence fondamentale est probablement ailleurs; elle n'est pas entre ceux qui savent et ceux qui doutent, mais entre ceux qui croient pouvoir déduire tout un corps de doctrine pour l'action à partir d'un nombre limité d'études scientifiques, et ceux qui savent devoir trouver des compromis entre un savoir encore très partiel et la validation par l'expérience de savoir-faire plus ou moins empiriques, dans le contexte forestier si particulier qui impose une certaine cohérence sur le long terme.

Les premiers sont prompts à sommer les forestiers de renoncer en bloc à certaines pratiques traditionnelles pourtant opérationnelles, pour adopter des conclusions découlant du dernier état des connaissances, en sous-estimant l'inertie des systèmes forestiers et la prudence indispensable des réorientations. Les seconds sont prompts à faire de pratiques héritées des tâtonnements de nos prédécesseurs une norme dont s'écarter conduirait inévitablement à l'échec. Si les forestiers ont parfois tendance à butiner dans l'état le plus récent des connaissances pour justifier des pratiques anciennes, certains militants écologistes maniant le registre scientifique ont parfois tendance à occulter toutes les incertitudes et les questions encore ouvertes, qui rendent légitimes d'autres approches possibles. Une telle attitude permet alors de dicter des solutions, en refusant d'avoir à les argumenter et justifier en détail, et d'écarter a priori les thèses d'autres experts partant d'autres prémices ou sensibles à d'autres analyses. L'insuffisance de dialogue et un certain manque d'intérêt pour une expérimentation raisonnable s'inspirant des derniers travaux scientifiques et des expériences étrangères conduisent paradoxalement à renverser les fronts. Dans le débat sur la gestion durable, les scientifiques ou ceux qui pensent se réclamer d'une véritable approche scientifique apparaissent souvent comme ceux qui savent et qui parlent catégoriquement, et les forestiers comme ceux qui doutent tellement de leurs connaissances que seules les "recettes" héritées de nos prédécesseurs peuvent être recommandées.

Sur un tout autre plan, le recours quasi systématique du ministère de l'Environnement à l'expertise scientifique bénévole, détenue très souvent par des naturalistes militant par ailleurs dans le monde associatif, pour mener des inventaires préparatoires à l'application d'une politique d'État (ZNIEFF, Directive Habitats,...) n'est pas sain, car il est ensuite très difficile de remettre en cause ne serait-ce qu'une partie d'une expertise bénévole dont le bon fonctionnement de l'administration dépend. II est par ailleurs très surprenant que la caution scientifique de ces inventaires soit apportée de façon collective par des comités, sans aucune identification publique du scientifique responsable de l'inventaire, en contradiction flagrante avec les règles ordinaires de la déontologie scientifique. Le dossier de mise en œuvre de la Directive Habitats en forêt ne peut que souffrir de cette situation qui rend toute contestation ou toute demande de révision "scientifiquement" suspecte, et "calomnieuse" pour ceux qui se sont dévoués bénévolement à un travail ingrat, sans moyens et dans des délais excessivement courts. Ce type de situation ne peut malheureusement qu'attiser les incompréhensions et les antagonismes entre les forestiers et les naturalistes du monde associatif. Par ailleurs il est parfois difficile à un homme de faire une distinction nette entre son activité scientifique, ses convictions éthiques, et son engagement militant dans le secteur de la protection de la nature, lorsqu'il est interpellé comme expert à la limite de son domaine de compétence. À ces multiples occasions se révèle clairement la question du rôle potentiellement trouble de l'expertise scientifique non régulée, tel que Dominique Bourg l'a mis en évidence dans son article.

- En règle générale, les forestiers redoutent le conflit, perçu comme un risque pour la continuité et la cohérence des actions, alors que les associations de protection de la nature le cherchent, car elles en ont besoin pour défendre publiquement leurs thèses, en l'absence de structures formelles de négociation permanente. Cette situation de faiblesse des forestiers tient en partie à leur culture d'ingénieur. Celle-ci privilégie trop souvent la résolution de problèmes, en sous-estimant l'effort indispensable à l'identification méthodique des problèmes, sans les circonscrire trop rapidement aux problèmes dont les termes techniques semblent maîtrisables. Les élèves-ingénieurs forestiers ont besoin d'être davantage formés au diagnostic de problèmes non circonscrits a priori, en déve- 
loppant leur sens de l'écoute fidèle (vérification que l'écouté se retrouve effectivement dans ce que retient l'écouteur) et en les faisant travailler sur l'identification de problèmes concrets, sans mettre en même temps la pression sur l'identification de solutions, ce qui fausse souvent toute la phase d'analyse et d'ècoute. Le schéma culturel dominant chez les ingénieurs forestiers européens et nordaméricains ne laisse en effet que très peu de place pour l'expression des divergences d'analyses et à peu près aucune pour la gestion des conflits.

Comme l'expliquait François Terrasson, du Muséum national d'Histoire naturelle, lors du forum international sur le développement durable, organisé par I'UNESCO en 1991, les conflits qui n'explosent pas ne se résolvent pas. Le conflit doit être ouvert, sinon on négocie sur autre chose. Le premier travail est donc de faire sortir les oppositions réelles dans toute leur ampleur. Mais, dans ce contexte conflictuel, le négociateur doit avoir un entraînement éprouvé à la pression émotionnelle, ce qui sera d'autant plus dur dans les affaires de nature où justement il s'investit passionnèment. Ceci est vrai pour le forestier comme pour l'écologiste. C'est en effet un des paradoxes du débat public sur la forêt car, parallèlement, pour être crédible devant des tiers, le forestier doit s'impliquer personnellement. "La présentation d'explications à un auditoire non concerné affectivement débouche sur le vide. Cette erreur s'explique aisément par l'analyse du profil psychologique du naturaliste. Exerçant une profession scientifique basée sur la rigueur, le spécialiste de la nature oublie vite que sa motivation première et permanente repose sur son émerveillement et sur un intérêt passionnel " (11). II est vrai que, derrière les discours d'expert, les forestiers dissimulent souvent une émotion ou une fascination devant les beautés de la forêt, à la source de leur vocation, mais qu'ils n'expriment publiquement que très peu ce registre émotionnel qui nourrit pourtant leur implication personnelle si forte dans leur métier ou leur propriété forestière.

Pour se faire entendre et comprendre de la société, les forestiers ont besoin de ne pas parler sur le seul registre de la rationalité, car le débat sur la gestion durable est loin d'être seulement un débat technique. L'oublier laisse le champ libre à des discours irrationnels et sèduisants qui opposent facilement la froideur rebutante du technicien et la chaleur sympathique de l'écologiste. Lorsque la télévision donne la parole successivement à un ingénieur forestier en costume-cravatte dans son bureau, puis à un écologiste en chemisette en forêt, qui ne perçoit pas l'opposition entre la technocratie et la proximité de la nature. Lorsque les forestiers privilégient trop souvent la parole des responsables hiérarchiques pour rẻpondre aux contestations de tous genres, ne perçoivent-ils pas que l'auditeur entend aussi l'opposition d'un discours d'institution et d'une parole libre, enracinée dans une spontanéité de terrain. Dans certains cas, les hommes que la société appelle encore les "gardes forestiers" ont une crédibilité a priori et une force de conviction plus forte que les ingénieurs forestiers. Mais jusqu'où ne pas aller trop loin dans le registre du savoir-faire de la communication, qui peut parfois déformer ou occulter les données d'un choix, afin de ne pas rentrer sur le terrain de la manipulation consciente de l'opinion publique ? Le débat sur la gestion durable passe nécessairement par le fait d'assumer ouvertement et sans malaise certains conflits, et donc de se donner les moyens de se faire entendre. Mais, dans ce débat, pour l'ensemble des parties en conflit, quels sont les moyens éthiquement acceptables?

- Derrière le débat sur la gestion durable des forêts apparaissent, avec plus ou moins de netteté, toute une serie de conceptions possibles de la nature et des relations souhaitables entre l'homme et la nature. Ceci déconcerte souvent les forestiers qui perçoivent généralement les enjeux techniques avant les enjeux éthiques. II appartient aux sociologues et aux philosophes d'aider les forestiers à y voir plus clair dans les implications de ces différentes visions, parfois empreintes d'une certaine misanthropie. L'article de Steven L. Yaffee a montré l'importance qu'une organisation

(11) TERRASSON (F.). - - Perception-Communication-Comportement, une application des sciences cognitives dans le domaine de la conservation de la nature et de l'environnement. - UNESCO, SHS/91/CONF/802/COL/3, 1991. - 25 p. 


\section{Ch. BARTHOD}

forestière doit attacher à comprendre en temps voulu les demandes diffuses de la société, pour adapter ses analyses et ses propositions. Les forestiers ont une "certaine culture de l'ordre" et éprouvent de la difficulté à discerner derrière les revendications parfois brouillonnes des nombreuses associations la légitimité d'un interlocuteur démocratique. Ils insistent à juste titre sur la nécessaire continuité de la gestion forestière, et donc sur le besoin de durer au-delà des modes temporaires. Mais, pour durer, il faut paradoxalement s'adapter et savoir répondre aux questions d'une époque particulière. L'histoire de la foresterie française entre 1860 et 1914 a montré qu'une telle stratégie n'était pas incompatible avec des projets à long terme.

Lorsque Patrick Blandin, du Muséum national d'Histoire naturelle, critique les conséquences que les forestiers français tirent de la maxime de Parade (imiter la nature, hâter son œuvre), il porte le fer sur un point sensible (12). L'imitation de la nature relève en effet pour les forestiers du domaine de l'inspiration, en même temps que de la réflexion d'une expérience séculaire sur ses réussites et ses échecs. Mais la compréhension de cette maxime suppose une référence, explicitement absente chez Parade mais pourtant fortement sous-jacente, à la légitimité de manipuler les écosystèmes forestiers au profit de l'homme (cf. la référence anthropomorphique à l'œuvre de la nature). Blandin insiste par contre exclusivement sur la conformité à la nature, et ironise sur la hâte des forestiers qui conduirait surtout à la simplification des écosystèmes, source de déboires; sa réflexion ne laisse pas de place évidente pour les droits et devoirs de l'homme qui cherche à satisfaire des besoins. Si les forestiers auraient bien tort de refuser toute pertinence aux critiques venant de l'étude du fonctionnement des écosystèmes subnaturels et de ne pas chercher à mieux définir les limites qu'ils ne veulent pas franchir dans la simplification des systèmes biologiques qu'ils gèrent, ils ne peuvent ressentir qu'un certain malaise devant des approches "chimiquement pures" de la forêt, où l'homme et la société sont absents. Or l'absence de référence à l'homme et à la société dans un projet forestier ne donne plus aucun contrepoids à la référence à la nature, ainsi promue à l'état de norme.

La compréhension de la nature qui semble dominante dans l'opinion publique relève davantage d'un conservatisme qui chercherait à perpétuer les pratiques forestières et les paysages d'avant une ère réputée prométhéenne, identifiée à la grande période d'action du Fonds forestier national (FFN). Dans tout conservatisme de ce type, se trouvent la sanction de certaines erreurs qu'il convient de corriger, mais aussi le refus d'un monde qui bouge, en oubliant que la "bonne" forêt d'avant 1945 reflétait aussi un équilibre écologique, économique et social instable et toujours en évolution. La tronçonneuse ne peut effacer la nostalgie de la hache, et les camions de grumes et de rondins celle des petits artisans de proximité. Ce refus est aussi celui de l'économique qui aurait contaminé et dégradé la forêt, depuis que les forestiers ont vraiment commencé à s'intéresser au secteur industriel à qui ils vendent des bois. Sans qu'il soit question de le réduire à cela, le débat sur la gestion durable joue profondément sur l'alliance possible entre écologie et conservatisme nostalgique.

- La question économique est incontournable, même si les forestiers n'ont cessé de se sentir mal à l'aise dans un cadre général façonné par les seules préoccupations du court terme. Dans une société démocratique, la possibilité existe de le déplorer et d'appeler de ses vœux une société autre, mais ceux qui ont en charge les arbitrages inhérents à toute gestion concrète d'un territoire particulier ne peuvent l'oublier. Par nature même, le monde scientifique et les associations de protection de la nature ne sont pas vraiment sensibles à cet aspect des choses, et il serait vain d'attendre de leur implication spontanée une réponse aux questions légitimes que se posent les gestionnaires forestiers dans le domaine économique, en un pays dont on a pu dire récemment (cf. Guy Sorman) que ses ressortissants étaient surdéveloppés en politique et sous-développés en économie. Ces questions ne s'appliquent d'ailleurs pas qu'aux seules modalités alternatives de gestion qui sont données en exemple par certains scientifiques et beaucoup d'associations de protection de la nature. En effet, certaines options sylvicoles actuelles suscitent également des interrogations de nature économique.

(12) Les forêts : développement ou conservation durable ? - Courrier de l'Environnement de IINRA, $\boldsymbol{n}^{\mathrm{C}}$ 25, 1995, pp. 47-52. 
Quoi qu'il en soit, sans sous-estimer les aides directes et indirectes apportées par l'État à la gestion forestière, la vente de produits marchands, répondant à une demande de la société, demeure encore aujourd'hui la solution la plus performante pour financer une gestion durable, dès lors qu'on exclut de confondre gestion durable et mise en réserve intégrale ou retour à une simple cueillette. Dans cette conception, il est par ailleurs légitime de chercher à agir sur les écosystèmes forestiers pour modifier, en qualité ou en quantité, les produits récoltés en forêt, en fonction des besoins humains. Dans son immense majorité, la forêt française est une forêt qui est ou qui a été cultivée, et lui appliquer les grilles d'analyse de la forêt naturelle est absurde. Mais, d'un autre côté, il serait dangereux de chercher à tracer une limite définitive entre une forêt subnaturelle et une forêt cultivée, en oubliant le continuum entre ces deux archétypes dans la situation française. Dans le débat parfois assez byzantin sur la gestion durable et l'exploitation des produits ligneux, il convient de revenir à des principes de base. La véritable limite se situe entre les forêts où l'action positive de l'homme est négligeable et où celui-ci se comporte en exploitant minier, et les forêts où l'homme prétend récolter les produits de sa gestion, "eu regard à ce que les dites forêts se puissent perpétuellement soutenir en bon état " (Ordonnance de Brunoy, 1346).

Les forestiers français ne peuvent que partager avec les écologistes une forte préoccupation devant les risques que représenterait l'importation croissante en Europe occidentale de produits ligneux issus de forêts non cultivées. Les produits venant d'une cueillette ou d'une exploitation minière, notamment de Russie (directement ou via des pays nordiques), sont en effet susceptibles de déstabiliser la majeure partie des marchés européens, actuellement approvisionnés essentiellement à partir de forêts tempérées cultivées. Protéger et valoriser les débouchés de nos forêts cultivées, c'est aussi contribuer à protéger les dernières forêts naturelles de pays qui cherchent prioritairement à financer leur développement économique et non à mettre en œuvre une gestion durable, compte tenu d'urgences sociales incontestables. II est nécessaire de créer des conditions qui permettent aux produits forestiers issus des forêts cultivées de conquérir ou reconquérir des marchés rémunérateurs, pour que le gestionnaire forestier puisse accepter de prendre en charge des contraintes environnementales supplémentaires. La conception des systèmes communautaires de normalisation et de certification jouera très certainement un rôle important de ce point de vue, négatif ou positif, alors que cette réflexion globale sur l'avenir des forêts cultivées n'est absolument pas prise en compte dans les réflexions de l'Union européenne.

Certes une plus grande prise en compte de toutes les interactions entre la sylviculture et le milieu (sols, eaux, espèces animales et végétales, paysages, ...) n'est pas systématiquement synonyme de coûts supplémentaires, et il conviendrait de commencer par bien établir ce qui relève d'une gestion normale avant de raisonner en surcoûts. Dans de nombreux cas, plus d'écologie passe par plus d'économique. Mais il est difficile de faire l'impasse sur l'évaluation économique des simples inflexions ou des modifications plus importantes de gestion. Le secteur aval doit en effet être en mesure de répercuter des coûts d'approvisionnement accrus lorsque les bois sont issus d'une gestion plus contraignante, faute de quoi les gestionnaires forestiers risquent d'emmener l'ensemble de la filière-bois dans une impasse. Au lieu de donner faussement l'impression de combattre la demande d'"écologie" au nom d'une prioritè absolue donnée à l'économie, les forestiers ont tout intérêt à lier publiquement les deux démarches. II serait de l'intérêt de l'Union européenne de le comprendre rapidement, et des pays de l'Union européenne de découvrir que ce qui les unit sur ce sujet est plus fort que les rivalités commerciales.

II faut en effet simultanément aller au devant des attentes de la société dans le domaine de la prise en compte de l'environnement dans la gestion forestière (dans des limites raisonnables), et consentir un effort considérablement accru pour mieux insérer le secteur économique du bois et du papier dans le contexte technologique, financier et commercial moderne. Ce dernier point passe notamment par une amélioration de la mobilisation des bois, par une meilleure organisation des marchés, et par un développement de la recherche industrielle pour préparer le bois à reconquérir des parts de 


\section{Ch. BARTHOD}

marché sur ses matériaux concurrents. Si un effort pédagogique doit être légitimement consenti pour expliquer aux citadins les multiples bénéfices environnementaux découlant d'une forêt variée en équilibre, il faut pouvoir réussir parallèlement à promouvoir efficacement le bois comme éco-matériau, et l'achat de produits en bois comme une réponse écologiquement responsable du consommateur à ses inquiétudes sur le monde de demain, et non comme un facteur de destruction de la forêt.

Si le climat devenait trop conflictuel, à l'intérieur de chaque pays et entre pays européens, ou si cette attention parallèle à l'environnement et à l'économie de la filière-bois n'arrivait pas à s'imposer, il serait à craindre que le débat sur la gestion durable des forêts tempérées ne conduise à détruire les efforts consentis au cours des dernières décennies pour promouvoir une meilleure compréhension mutuelle des objectifs et contraintes respectifs de la gestion forestière et des industries valorisant les produits forestiers. Dès lors, au lieu d'être une chance pour la forêt française, le débat sur la gestion durable aurait été le cimetière d'une certaine conception française (mais aussi en grande partie européenne) de la foresterie, dont certains de nos partenaires et concurrents commerciaux tireraient profit, au moins dans un premier temps. Entendre certains discours écologistes (américains, mais aussi européens et même français) plaider en faveur du béton, du PVC et de l'aluminium contre le bois, ressource renouvelable dont les conditions de "production" peuvent s'adapter aux demandes nouvelles, ne peut que susciter un vif malaise, même si ces discours s'abritent derrière l'apparente neutralité de bilans énergétiques dont les a priori ne sont pas explicités.

$$
\text { 㠑 }
$$

Ces difficultés et questions troubles ne doivent en aucune façon servir de justification aux forestiers pour se mettre en réserve des réflexions et des conflits sur la gestion durable des forêts tempérées. Il est encore temps pour eux de s'engager résolument dans ces nouveaux débats publics, avec leurs convictions mais aussi une certaine humilité face à l'évolution des connaissances et des attentes de la société, et de gagner cette bataille de la gestion durable, en collaboration avec tous ceux qui sont prêts à se mobiliser sur cette question, d'une manière qui soit à la fois pertinente au regard des défis actuels et fidèle au meilleur de nos traditions forestières. C'est la meilleure contribution que les forestiers peuvent apporter à la cause d'un développement durable des sociétés dont ils sont citoyens et vis-à-vis desquelles ils sont comptables de leurs choix de gestion.

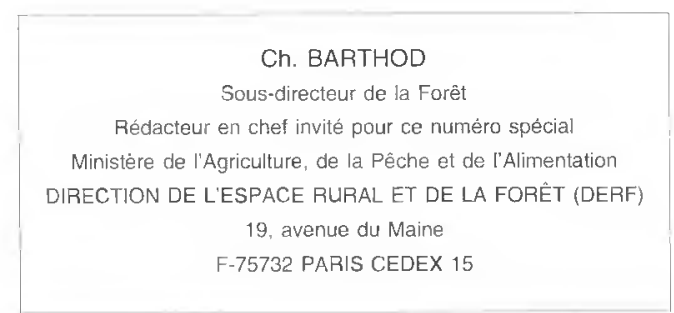

